predicate stent retriever technologies. We report the results of the Vesalio Neva VS for the Treatment of Symptomatic Cerebral Vasospasm Following Aneurysmal Subarachnoid Hemorrhage (VITAL) Study.

Methods This is a single arm, prospective, multi-center trial to assess the safety and probable benefit of the Neva VS device to treat symptomatic vasospasm. Patient were screened and enrolled if they had confirmed cerebral vasospasm $\geq 50 \%$ on non-invasive imaging and confirmed on digital subtraction angiography. Patient's who failed medical therapy of systemic hypertension and oral nimotop and a vessel diameter between 2.0 - $4.0 \mathrm{~mm}$ were considered. Patients were excluded with a Hunt-Hess Grade 5 or large core infarctions with an ASPECTS score $\leq 5$ prior to treatment. The Neva VS device was deployed for an average of 5 minutes in the target vessel and vessel diameters were measured pre and post treatment by an independent core lab. The primary endpoint was achieving a $>50 \%$ vessel diameter after treatment with the Neva VS device.

Results A total of 30 patients with a mean age of $52.3 \pm$ 11.1 years and mean Hunt-Hess grade of $3.1 \pm 0.9$ were enrolled in the study. The majority of patients were women 86.7\% ( $\mathrm{N}=26)$ who had undergone coiling 93.3\%(N=28). A total of 35 procedures were performed with 28 patients undergoing one treatment, two patients with two treatments, and one patient with three treatments. A total of 72 vessels were treated in theses 35 procedures. The breakdown by vessel is as follows: MCA 40 (55.5\%), ICA 17 (23.6\%), ACA 8 $(11.1 \%)$, posterior circulation $7(9.7 \%)$. The mean narrowing of the target vessel pretreatment with $>50 \%$ luminal narrowing after core lab adjudication $(\mathrm{N}=66)$ was $68.4 \%$ with reduction of the narrowing to $30.6 \%$ post treatment. The primary endpoint was achieved in 56 of 66 segments (84.8\%). Two patients $(6.6 \%)$ developed a transient cerebral thrombus during the procedure without neurological sequelae. Two patients (6.6\%) suffered a vessel rupture from balloon angioplasty and unrelated to the Neva VS treatment.

Conclusions The Neva VS device appears safe and effective at reducing luminal compromise related to cerebral vasospasm following aneurysmal subarachnoid hemorrhage. This treatment offers a novel strategy with more operator control and lower risk of vessel perforation.

Disclosures R. Gupta: 1; C; Stryker Neurovascular PI ASSIST Registry, Zoll PI RECCLAIM II (No compensation), Cerenovous Steering Committee MEMBRANE study, Medtronic Steering Committee ELEVATE Study, Penumbra CEC MIND Trial, Vesalio PI CLEAR Study, Rapid Medical PI Tiger Study. K. Woodward: 2; C; Cerenovous, Medtronic, Microvention, Penumbra, Stryker. D. Fiorella: 1; C; Cerenovous, Medtronic, Microvention, Penumbra, Stryker. 2; C; Penumbra, Stryker. 4; C; Penumbra. H. Woo: 4; C; Vascular Simulations. D. Liebeskind: 2; C; Cerenovous, Rapid Medical, Genentech, Medtronic, Stryker. D. Frei: 2; C; Medtronic, Penumbra. A. Siddiqui: 2; C; Amnis, Apellis, Boston Scientific, Canon Medical Systems, Cerebrotech Medical System, Cerenovous, Corindus, Endostream Medical, Imperative Care, Integra Life Sciences, Medtronic, Microvention, Minnetronix Neuro, Penumbra, PerFlow, Rapid Medical, Q'Apel Medical, Rebound Therapeutics Corp, Serenity Medical, Silk Road Medical, StimMed, Stryker, Three Rivers Medical, VasSol, Viz.ai, Gore Associates. 6; C; Cognition Medical, Amnis, Blink IT Technologies, Adona Medical, Bend IT Technologies, Cardinal Consultants, Cerebrotech Medical System, Imperative Care, Instylla Inc, International Medical Distribution
Partners, IRRAS, Q’Apel, Perflow Medical, Rebound Therapeutics Corp, Neurovascular Diagnostics Inc, Radical Catheter Technologies. R. DeLeacy: 1; C; Medtronic, Asahi Inc. 2; C; Cerenovous, Q’Apel Medical Inc., Penumbra, Imperative Care, Mivi Neurosciences. R. Hanel: 2; C; Rapid Medical, Stryker, Medtronic, Balt, Phenox, Elum, MIVI, ThrombX, Endostream, RIST, REIST, Serenity, BendIT. A. Maud: None.

\section{P-045 NON-DEFINITIVE SURGICAL CLIPPING OF CEREBRAL ANEURYSMS: A META-ANALYSIS OF WRAPPING, RESIDUAL, AND RECURRENCE RATES}

${ }^{1} \mathrm{D}$ Schartz*, ${ }^{2} \mathrm{~T}$ Mattingly, ${ }^{2} \mathrm{~T}$ Bhalla, ${ }^{2} \mathrm{M}$ Bender. ${ }^{1}$ Department of Imaging Sciences, University of Rochester Medical Center, Rochester, NY; ${ }^{2}$ Department of Neurosurgery, University of Rochester Medical Center, Rochester, NY

\subsection{6/neurintsurg-2021-SNIS.81}

Introduction Microsurgery for cerebral aneurysms is called definitive, yet some patients undergo a craniotomy that results in incomplete treatment. Three types of incomplete treatment are: aneurysm wrapping, post-clipping remnants, and late regrowth of obliterated aneurysms. Each of these is associated with increased risk of subarachnoid hemorrhage. This was a systematic review and meta-analysis to quantify the overall rate of non-definitive open surgery for cerebral aneurysms.

Methods A comprehensive literature review using MEDLINE and Cochrane Library databases up to February of 2021 was completed. The following search terms were used: (Intracranial Aneurysm) AND (Clip) AND (residual), (Intracranial Aneurysm) AND (Clip) AND (recurrence), (Intracranial Aneurysm) AND (Wrapping), (Intracranial Aneurysm) AND (Regrowth). Articles were screened based on specific inclusion criteria for each respective analysis. We included studies of aneurysms in a single location (e.g. middle cerebral artery) but excluded studies limited to morphological sub-types or sizes (e.g. fusiform or giant aneurysms). Pooled rates of aneurysm wrapping, residua confirmed by imaging, and regrowth following confirmed total occlusion were subsequently calculated along with corresponding confidence intervals using a random effects model. An assessment of statistical heterogeneity and publication bias among the included studies for each analysis was also completed with resultant $\mathrm{I}^{2}$ and Egger's test P-values.

Results A total of 812 unique studies were identified by literature review and 65 studies met inclusion criteria for final analysis. In 43 studies that met inclusion criteria, 573/15,715 aneurysms were wrapped for a rate of 3.3\% (95\% Confidence Interval [CI], $2.7 \%$ to $4.0 \%$ ). In 44 studies, 912/14,045 aneurysms had residual neck or dome filling for a rate of $6.3 \%$ (95\% CI, $5.1 \%$ to $7.5 \%$ ). In 15 studies, $71 / 2,568$ originally occluded aneurysms showed regrowth for a rate of $2.1 \%$ (95\% CI, $1.2 \%$ to $3.1 \%$ ). Together, there was a total rate of non-definitive treatment of $11.7 \%$ (95\% CI, $11.1 \%$ to $12.3 \%$ ) for open surgery of cerebral aneurysms. $\mathrm{I}^{2}$ values for the wrapping, residual, and regrowth analyses were 88\%, 93\%, and $58 \%$, respectively, indicating significant heterogeneity among the included studies. Egger's test P-value for the wrapping, residual, and regrowth analyses were $0.55,0.24$, and 0.13 , respectively, suggesting no significant publication bias among the included studies. Sub-analyses revealed that the rate of aneurysm wrapping has decreased over time based on publication date, while rates of residua and regrowth have not 
changed. Rates of wrapping, residua, and regrowth were not statistically different based on aneurysm rupture status.

Conclusion Open surgery for cerebral aneurysm results in incomplete treatment approximately $12 \%$ of the time. This should be taken into account when considering endovascular verses open surgical therapy and counseling patients. These results should be interpreted with caution considering the significant amount of heterogeneity between the included studies.

Disclosures D. Schartz: None. T. Mattingly: None. T. Bhalla: None. M. Bender: None.

\section{P-046 FACTORS ASSOCIATED WITH INCOMPLETE OCCLUSION OF INTRACRANIAL ANEURYSMS AT FOLLOW UP AFTER TREATMENT WITH WOVEN ENDOBRIDGE (WEB) DEVICE}

K Javed ${ }^{*}$, A Fortunel, N Haranhalli, D Altschul. Neurosurgery, Montefiore Medical Center, Bronx, NY

\subsection{6/neurintsurg-2021-SNIS.82}

Objective The Woven EndoBridge (WEB) device is a novel treatment option for wide-necked bifurcation intracranial aneurysms (WBNA), which historically were a challenge to treat using conventional endovascular therapies. According to the WEB-IT study which led to the WEB device gaining FDA approval in the US, the complete aneurysmal occlusion rate is $53.8 \%$ while the adequate occlusion rate is $84.6 \%$ at one year follow up. While this device has had good results, there remains a subset of WBNA that fail this treatment. The main objective of this study is to identify factors that are associated with incomplete occlusion of WEB treated aneurysms at follow up.

Methods This is a retrospective study of forty-one patients with intracranial aneurysms who were treated with WEB placement at a single institution since 2019. Data was collected via electronic medical record (EMR) review on patient demographics, medical comorbidities, aneurysm characteristics, procedural details, and occlusion status at six months follow up. Bivariate analyses were performed comparing completely occluded aneurysms with incompletely occluded aneurysms such as neck remnants and residual aneurysms.

Results Of the 41 patients, follow up data was only available for 25 patients. $13(52 \%)$ of those 25 patients had completely occluded treated aneurysms while $8(32 \%)$ patients had a neck remnant and $4(16 \%)$ patients had a residual aneurysm at six months follow up. On bivariate analysis, patients with a neck remnant were found to have a median angle of $29.7^{\circ}$ in the true neck view compared to a median angle of $17.7^{\circ}$ in patients with complete occlusion $(p$ value $=0.050)$. In patients with residual aneurysms, the median angle in the down the barrel view was $9.3^{\circ}$ while it was $34.2^{\circ}$ in patients with occluded aneurysms ( $p$ value $=0.023$ ). Furthermore, the average diameter of the WEB device deployed was $7.5 \mathrm{~mm}$ in patients with residual aneurysms compared to $6.0 \mathrm{~mm}$ in the complete occlusion group ( $\mathrm{p}$ value $=0.026$ ). Lastly, patients with residual aneurysms were more likely to have a longer procedure time $(p$ value $=0.027$ ) and less likely to have an immediate angiographic outcome of complete occlusion ( $p$ value $=0.006$ ) .

Conclusion The angular measurements of the aneurysms along with size of the WEB device used, procedure length and immediate post-treatment occlusion status may predict the occlusion status of the treated aneurysm at six months follow up.

Disclosures K. Javed: None. A. Fortunel: None. N. Haranhalli: None. D. Altschul: None.

\section{P-047 MULTIMODAL ENDOVASCULAR TREATMENT OF WIDE- NECKED ANEURYSMS: SAFETY AND OCCLUSION OUTCOMES FROM THE STERLING REGISTRY}

${ }^{1} \mathrm{R}$ De Leacy* ${ }^{*}{ }^{2} \mathrm{~A}$ Puri, ${ }^{3} \mathrm{R}$ Starke, ${ }^{4} \mathrm{~B}$ Jankowitz, ${ }^{5} \mathrm{~A}$ Yoo, ${ }^{6} \mathrm{G}$ Pero, ${ }^{7} \mathrm{C}$ Chivot, ${ }^{8} \mathrm{~T}$ Yao, ${ }^{9} \mathrm{O}$ Zaidat. ${ }^{1}$ Neurosurgery, Mount Sinai Health System, New York, NY; ${ }^{2}$ Radiology, Neurological Surgery, and Neurosurgeny, UMass Memorial Health Care, Boston, MA; ${ }^{3}$ Neurosurgery, UMiamilJackson Memorial, Miami, FL; ${ }^{4}$ Neurological Institute, Neurological Surgery, and Stroke Program, Cooper University Health Care, Pittsburgh, PA; ${ }^{5}$ Neurological Surgery, Texas Stroke Institute, Plano, TX; ${ }^{6}$ Neuroradiology, Ospedale Niguarda, Milan, Italy;

${ }^{7}$ Radiology, CHU Amiens Picardie, Amiens, France; ${ }^{8}$ Neurosurgery, Norton Neuroscience Institute, Norton Healthcare, Louisville, KY; ${ }^{9}$ Neuroscience Institute, Mercy St. Vincent Hospital, Toledo, $\mathrm{OH}$

\subsection{6/neurintsurg-2021-SNIS.83}

Purpose We present core-lab adjudicated immediate post-procedural angiographic outcomes following endovascular treatment of side wall and bifurcation wide-necked aneurysms using MICRUSFRAME and GALAXY coils and various adjunctive devices within the STERLING registry.

Methods STERLING is an ongoing single-arm prospective post-market surveillance registry evaluating ruptured/unruptured aneurysms treated with MICRUSFRAME and GALAXY coils (Cerenovus, Irvine, CA). All cases of wide-neck aneurysm treatment (neck $\geq 4 \mathrm{~mm}$ or dome-to-neck ratio $<2$ ) were extracted from the registry. Subgroups were defined according to the adjunctive devices (if any) used during treatment. The primary outcome measures were core-lab adjudicated modified Raymond-Roy (mRR) occlusion at final procedural angiogram, and procedure-related adverse events.

Results The study cohort contained 168 patients treated for 168 wide-necked aneurysms. The average age was 59.1 (SD 10.5 ), and $71.4 \%$ of patients were female. Previously treated aneurysms accounted for $9.5 \%$ of the cohort, and $45(26.8 \%)$ were acutely ruptured. All acutely ruptured aneurysms were treated with coiling alone. One hundred thirty-seven aneurysms $(81.5 \%)$ were located in the anterior circulation, and 90 $(53.6 \%)$ were located at a bifurcation point. With regard to size, $22.6 \%$ of aneurysms were $<5 \mathrm{~mm}$ in diameter, $71.4 \%$ were $\geq 5 \mathrm{~mm}$ and $<13 \mathrm{~mm}$, and the remaining $6.0 \%$ were $\geq$ $13 \mathrm{~mm}$. The average procedure time was 1.74 hours (SD 0.99 ) and the average fluoroscopy time was 49.7 minutes (SD 29.6).

Aneurysms were treated using the following modalities: coiling alone (58.3\%), stent-assisted coiling (24.4\%), coiling plus flow diversion (8.3\%), Pulserider-assisted coiling (4.2\%), balloon-assisted coiling (1.2\%), other adjunctive devices (1.8\%), and multiple adjunctive devices (1.8\%). Across the entire cohort, $82.1 \%$ of patients achieved adequate occlusion, defined as mRR class I or II. Angiographic outcomes according to treatment modality are summarized in table 1.

Procedure-related adverse events occurred in 7 cases (4.2\%). These included 3 intraprocedural ruptures (1 ruptured, 2 unruptured at baseline), 1 cerebrovascular accident, and 3 asymptomatic thromboembolic events. There were no deaths. Conclusion Endovascular treatment of wide-neck aneurysms using MICRUSFRAME and GALAXY coils is safe and effective 